\title{
Atmospheric Biomarkers and their Evolution over Geological Timescales
}

\author{
Lisa Kaltenegger ${ }^{1} \dagger$, Jucks K. ${ }^{1}$ and Traub W..$^{1,2}$ \\ ${ }^{1}$ Harvard-Smithsonian Center for Astrophysics, 60 Garden St, Cambridge, MA 02138, USA \\ email: lkaltenegger@cfa.harvard.edu; kjucks@cfa.harvard.edu \\ ${ }^{2}$ Jet Propulsion Lab, M/S 301-451, 4800 Oak Grove Dr., Pasadena CA, 91109, USA \\ email: wtraub@jpl.nasa.gov
}

\begin{abstract}
The search for life on extrasolar planets is based on the assumption that one can screen extrasolar planets for habitability spectroscopically. The first space born instruments able to detect as well as characterize extrasolar planets, Darwin and terrestrial planet finder (TPF-I and TPF-C) are scheduled to launch before the end of the next decade. The composition of the planetary surface, atmosphere, and its temperature-pressure profile influence a detectable spectroscopic signal considerably. For future space-based missions it will be crucial to know this influence to interpret the observed signals and detect signatures of life in remotely observed atmospheres. We give an overview of biomarkers in the visible and IR range, corresponding to the TPF-C and TPF-I/DARWIN concepts, respectively. We also give an overview of the evolution of biomarkers over time and its implication for the search for life on extrasolar Earth-like planets. We show that atmospheric features on Earth can provide clues of biological activities for at least 2 billion years.
\end{abstract}

Keywords. astrobiology, astrochemistry, extrasolar planets, Earth, atmospheric biomarkers.

\section{Introduction}

Indirect methods to detect extrasolar terrestrial planets such as radial velocity, astrometry, and transits only allow getting morphological information. The planet is deduced from its effect on the parent star under survey. To understand the properties of a planet we need to collect photons from the planet directly to determine its physical and chemical properties. Spectral analysis of extrasolar planets is a very young science that only recently achieved first results for hot Jupiters. Some atmospheric components like Na were detected in absorption in the upper atmosphere of HD209458b (Charbonneau, Brown, Noyes et al. (2002)). The Spitzer telescope recently detected the infrared emission from two transiting hot Jupiters by subtracting the stellar flux when the transiting planet is eclipsed by its star from the combined flux of the star and planet (see Deming, Seager, Richardson et al. (2005), Charbonneau, Allen, Megeath et al. (2005)).

The first space born instruments able to detect as well as characterize extrasolar planets are DARWIN (see e.g. Kaltenegger, Fridlund \& Karlsson (2005)), TPF-I and TPF-C (see e.g. Borde \& Traub (2005)). DARWIN/TPF-I are both based on the concept of a free flyer IR interferometer architecture, and TPF-C is envisioned as a coronagraph in the visible. The strategy to search for biological activity on terrestrial planets is based on the assumption that one can use spectroscopy to screen extrasolar planets for habitability. Basing the search for life on the carbon chemistry assumption allows establishing

$\dagger$ Present address:Harvard-Smithsonian Center for Astrophysics, MS20, 60 Garden Street, Cambridge, MA 02138, USA 
criteria for habitable planets in terms of their size and distance from their stars (Kasting, Whitmire \& Reynolds (1993)). Note that observations over interstellar distances can only distinguish those planets whose habitability and biological activity is apparent from observations of the reflected or emitted radiation. A crucial factor in interpreting planetary spectra is the point in the evolution of the atmosphere and its biomarkers over time. Concentrating on the evolution of our planet we establish a model for its atmosphere and the detectable biomarkers over its evolution history. Figure 2 shows that atmospheric features on Earth can provide clues of possible life forms for at least 2 billion years (see Kaltenegger, Traub \& Jucks (2005)).

\subsection{Atmosphere evolution on Earth}

The Earth formed about 4.5 billion years ago. The primitive atmosphere was formed by the release of volatiles from the interior, and/or volatiles delivered during the late bombardment period. This atmosphere was most likely dominated by carbon dioxide, with nitrogen being the second most abundant gas and trace amounts of methane, ammonia, sulphur dioxide, hydrochloric acid and oxygen (see Kasting \& Siefert (2002)). Carbon dioxide and/or methane played a crucial role in the development of an early greenhouse effect that counteracted the lower solar output. Two major processes have changed the primitive atmosphere, the reduction of $\mathrm{CO}_{2}$ and the increase of $\mathrm{O}_{2}$. A huge amount of $\mathrm{CO}_{2}$ must have been removed from the atmosphere, most likely by the burial of carbon into carbonate rocks, though the process is still debated. Primitive cyanobacteria are believed to have produced oxygen. After most of the reduced minerals were oxidized, about two billion years ago, atmospheric oxygen could accumulate. Different schemes have been suggested to quantify the rise of oxygen and the evolution of life by anchoring the points in time to fossil finds (see e.g. Owen (1980) Schopf (1993) Ehrenfreund \& Charnley (2003)). There are still many open questions. We use a climate model based on work by Kasting \& Catling (2003), Pavlov, Hurtgen, Kasting, Arthur (2003), Segura, Krelove, Kasting et al. (2003) and Traub \& Jucks (2002) to create a schematic atmospheric model of our Earth over geological timescales. We find a surface temperature above the freezing point for all of Earth's history (not considering short term atmospheric changes during glaciation periods). Our radiative transfer model is based on the model by Traub \& Jucks (2002) that was developed to successfully analyse data from stratospheric balloon flight campaigns ongoing during the last few years. The same radiative transfer code was used to successfully analyse data from Earthshine measurements in the visible (400 to $900 \mathrm{~nm}$ ) (see Woolf, Smith, Traub et al.) and mid-infrared (700 to $2400 \mathrm{~nm}$ ) (see Turnbull, Traub, Jucks et al. (2005)). It shows excellent agreement with measurements in both the visible and IR wavelength band. We present the spectra of Earth over geological timescale in anticipation of proposed space based terrestrial planet search missions to operate between 500 to $1100 \mathrm{~nm}$ in the visible to mid-IR range and 6 to $20 \mu \mathrm{m}$ in the IR. Our model shows a considerable difference in biosignatures that can be detected with those missions. That should be able to constrain an Earth-like planet to its time in evolution.

\subsection{Atmospheres and Biomarkers}

The spectral characteristics of a planet are a very important guide to identifying the best wavelength region to probe for a planet as well as characterize the spectra once it has been detected. The TPF Science Working Group (TPF-SWG) identified the waveband between $8.5 \mu \mathrm{m}$ to $20 \mu \mathrm{m}$ and preferably $7 \mu \mathrm{m}$ to $25 \mu \mathrm{m}$, respectively, for the search for biomarkers in the mid-IR region and $0.7 \mu \mathrm{m}$ to $1.0 \mu \mathrm{m}$ and preferably $0.5 \mu \mathrm{m}$ to $1.1 \mu \mathrm{m}$ for the visible to near IR region (see Des Marais, Harwit, Jucks, et al. (2002)). The 
DARWIN project concentrates on the waveband between $6 \mu \mathrm{m}$ to $20 \mu \mathrm{m}$ (see Fridlund, Kaltenegger, et al. (2006)). In the thermal part of the spectrum, the shape gives a measure of the temperature of the object examined. Observations from $8 \mu \mathrm{m}$ to $12 \mu \mathrm{m}$ of the $\mathrm{H}_{2} \mathrm{O}$ continuum allow estimations of the surface temperature of Earth-like planets. For higher concentrations of water vapor in the atmosphere, the continuum gets lowered due to water absorption.

Biomarkers are features whose presence or abundance requires a biological origin (see Des Marais, Harwit, Jucks, et al. (2002), Meadows et al. (2005)). They are created either during the acquisition of energy and/or the chemical ingredients necessary for biosynthesis (e.g. atmospheric oxygen and methane) or are products of the biosynthesis (e.g. complex organic molecules and cells). As signs of life in themselves $\mathrm{H}_{2} \mathrm{O}$ and $\mathrm{CO}_{2}$ are secondary in importance because although they are raw materials for life, they are not unambiguous indicators of its presence. Taken together with molecular oxygen, abundant $\mathrm{CH}_{4}$ can indicate biological processes. Depending on the degree of oxidation of a planet's crust and upper mantel non-biological mechanisms can also produce large amounts of $\mathrm{CH}_{4}$ under certain circumstances. Oxygen is a chemically reactive gas. Reduced gases and oxygen have to be produced concurrently to be detectable in the atmosphere, as they react rapidly with each other. The $9.6 \mu \mathrm{m} O_{3}$ band is highly saturated and is thus a poor quantitative indicator, but an excellent qualitative indicator for the existence of even traces of $\mathrm{O}_{2}$. Ozone is a very nonlinear indicator of $\mathrm{O}_{2}$ because the ozone column depth remains nearly constant as $\mathrm{O}_{2}$ increases from 0.01 present atmosphere level (PAL) to 1 PAL.

$\mathrm{N}_{2} \mathrm{O}$ is a very interesting molecule because it is produced in abundance by life but only in trace amounts by natural processes. There are no $N_{2} O$ features in the visible and two weak $\mathrm{N}_{2} \mathrm{O}$ features in the IR. In the IR it can only be detected in regions strongly overlapped by $\mathrm{CH}_{4}, \mathrm{CO}_{2}$ and $\mathrm{H}_{2} \mathrm{O}$, so it is unlikely to become a prime target for the first generation of space-based missions searching for extrasolar planets that will work with low resolution, but it is an excellent target for follow up missions. Spectral features of $\mathrm{N}_{2} \mathrm{O}$ become apparent in atmospheres with less $\mathrm{H}_{2} \mathrm{O}$ vapor. In the mid-IR $\mathrm{N}_{2} \mathrm{O}$ has a band at $7.9 \mu \mathrm{m}$, comparable in strength to the adjacent $\mathrm{CH}_{4}$ band but weak compared with the overlapping $\mathrm{H}_{2} \mathrm{O}$ band. The absorption bands of those three species are different. It is not readily separable for low resolution spectroscopy for current Earth, but the methane feature is easily detectable for early type Earth planets according to our models. To detect and study surface properties we can only use wavelengths that penetrate to the planetary surface. On a cloud-free Earth, the diurnal flux variation caused by different surface features rotating in and out of view could be high. When the planet is only partially illuminated, more concentrated signal from surface features could be detected as they rotate in and out of view. Most surface features like ice or sand show very small or very smooth continuous opacity changes with wavelength. Figure 1 shows that the signal detected from a cloud free planet is lower than that from an Earth-like planet. Thus the integration time needed to detect such planets will be longer.

\subsection{The red edge of land planets}

An interesting example for surface biomarkers on Earth is the red edge signature from photosynthetic plants at about $750 \mathrm{~nm}$. Photosynthetic plants have developed strong infrared reflection as a cooling mechanism to prevent overheating and chlorophyll degradation. The primary molecules that absorb the energy and convert it to drive photosynthesis $\left(\mathrm{H}_{2} \mathrm{O}\right.$ and $\mathrm{CO}_{2}$ into sugars and $\left.\mathrm{O}_{2}\right)$ are chlorophyll A $(0.450 \mu \mathrm{m})$ and B $(0.680 \mu \mathrm{m})$. Several groups have measured the integrated Earth spectrum via the technique of Earthshine, sunlight reflected from the "dark" side of the moon. Earthshine measurements 

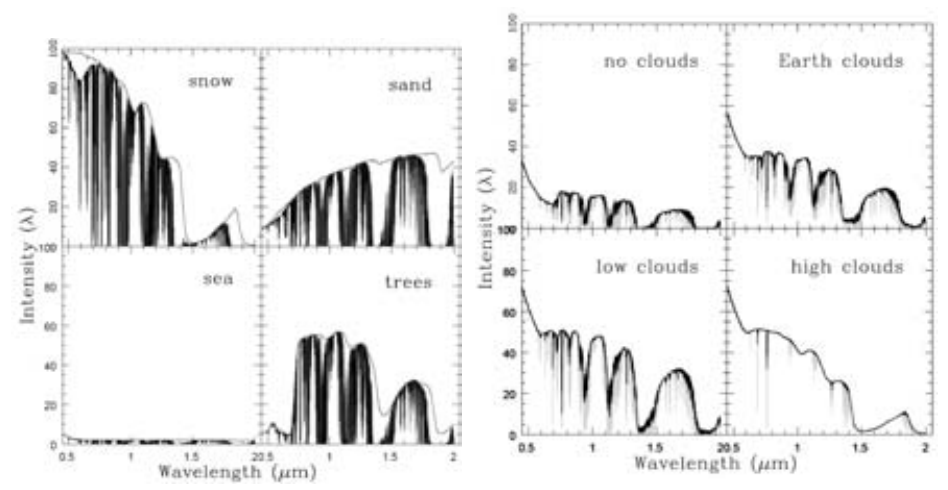

Figure 1. (left) Signal of a current Earth atmosphere considering surface features assuming a clear atmosphere without clouds; Different surface composition (a) snow (b) sea (c) trees (d) sand. (right) Signal of current Earth with realistic surface features and (a) no clouds, (b) $100 \%$ cumulus cloud coverage at $4 \mathrm{~km}$ and (c) $100 \%$ cirrus cloud coverage at $12 \mathrm{~km}$ and (d) a mixture of clouds resembling the present Earth.

have shown that detection of Earth's vegetation-red edge (VRE) is feasible but made difficult owing to its broad, essentially featureless spectrum and cloud coverage. Trying to identify such weak, continuum-like features at unknown wavelengths in an extrasolar planet spectrum requires models for different planetary conditions. Our knowledge of different surface reflectivities on Earth, like deserts, ocean and ice, help assigning the VRE of the Earthshine spectrum to terrestrial vegetation (see Figure 2). Earth's hemispherically integrated vegetation red-edge signature is weak, but planets with different rotation rates, obliquities, land-ocean fraction, and continental arrangement may have lower cloud-cover and higher vegetated fraction (see e.g. Seager (2002)).

\subsection{Model results}

Our calculations (see Kaltenegger, Traub \& Jucks (2005)) show that the composition of the surface (especially in the visible), the atmospheric composition and temperaturepressure profile can all have a significant influence on the detectability of a signal. The reflectivity of the Earth has not been static throughout the past $4.5 \mathrm{Ga}\left(\mathrm{Ga}=10^{9}\right.$ years ago). Oxygen and ozone became abundant about roughly $2.3 \mathrm{Ga}$, affecting the atmospheric absorption component of the reflection spectrum. About 2Ga, a green phytoplankton signal developed in the oceans and about $0.44 \mathrm{Ga}$, an extensive land plant cover developed, generating the red chlorophyll edge in the reflection spectrum. The oxygen and ozone absorption features could have been used to derive the presence of biological activity on Earth anytime during the past $50 \%$ of the age of the solar system, while the chlorophyll red-edge reflection feature evolved during the most recent $10 \%$ of the age of the solar system. Figure 1 shows the spectral signature of an Earth atmosphere assuming different surface composition and the big impact clouds can have on the spectrum. From the 4 surfaces plotted on the left one sees that an Earth-size ocean planet will be more difficult to detect than a snow covered planet due to its low albedo. The smooth lines in the left panel of Figure 1 show the albedo of the surfaces without overlayed atmosphere. The panel on the right in Figure 1 shows the strong impact clouds can have on the detected signal. Present Earth has about $60 \%$ cloud coverage. $100 \%$ cumulus (low) and $100 \%$ cirrus (high) cloud coverage, respectively, are shown for reference. A planet without clouds or snow has a lower overall albedo and thus requires a longer integration time for detection than a planet with clouds. Figure 2 shows Earth's reflected and thermal emission spectra respectively with its major molecular species $\left(\mathrm{H}_{2} \mathrm{O}, \mathrm{O}_{3}, \mathrm{O}_{2}, \mathrm{CH}_{4}, \mathrm{CO}_{2}\right.$, 


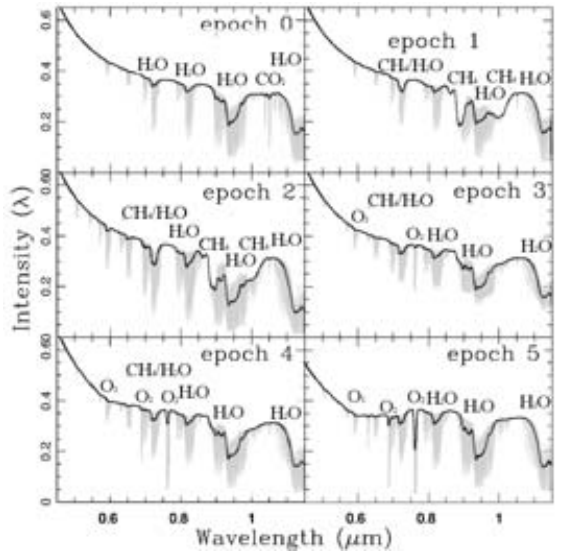

Figure 2. The atmospheric features siderably over its evolution from a current day atmosphere (epoch $5=$ present Earth) over a $\mathrm{CO}_{2} / \mathrm{CH}_{4}$-rich atmosphere (epoch 3) to a $\mathrm{CO}_{2}$ rich $(3.9 \mathrm{Ga}$ ago $=$ epoch 0 ) in both the visible (left) and the IR range (right). The black lines show spectral resolution of 70 for the visible and 25 for the IR, comparable to the proposed mission concepts.

$\left.\mathrm{N}_{2} \mathrm{O}\right)$ as well as minor contributors $\left(\mathrm{H}_{2} \mathrm{~S}, \mathrm{SO}_{2}, \mathrm{NH}_{3}, \mathrm{SF}_{6}\right.$, CFC-11, CFC-12). The dark lines show a resolution of 70 in the visible and 25 in the IR, as proposed for the TPF$\mathrm{C}$ and Darwin/TPF-I mission, respectively. The atmospheric features on an Earth-like planet change considerably over its evolution from a current day atmosphere (epoch5 = present Earth) over a $\mathrm{CO}_{2} / \mathrm{CH}_{4}$-rich (epoch 3 around $\left.2 \mathrm{Ga}\right)$ to a $\mathrm{CO}_{2}$ rich $(3.9 \mathrm{Ga}=$ epoch 0) atmosphere. $\mathrm{O}_{3}$ shows a strong feature in the IR for the last $2 \mathrm{Ga}$ of Earth's history even at a low resolution of $25 . \mathrm{O}_{2}$ shows a weaker feature in the visible for the last $2 \mathrm{Ga}$ of Earth's history at a resolution of 70 (note that no noise level is assumed for these calculations).

In the mid-IR the classical signatures of biological activity are the $9.6 \mu \mathrm{m} O_{3}$ band, the $15 \mu \mathrm{m} \mathrm{CO}$ band and the $6.3 \mu \mathrm{m} \mathrm{H}_{2} \mathrm{O}$ band or its rotational band that extends from $12 \mu \mathrm{m}$ out into the microwave region (Selsis, Despois, Parisot (2002)). For the visible, a similiar investigation has not been conducted yet. In the same spectral region, the $7.7 \mu \mathrm{m}$ band of $\mathrm{CH}_{4}$ is a potential biomarker for early-Earth type planets. In the visible to near-IR one can see a strong $\mathrm{O}_{2}$ absorption feature at $0.76 \mu \mathrm{m}$, a broadband $\mathrm{O}_{3}$ absorption at $0.45 \mu \mathrm{m}$ to $0.75 \mu \mathrm{m}$ and a strong $\mathrm{H}_{2} \mathrm{O}$ band at $0.94 \mu \mathrm{m}$. The strongest $\mathrm{O}_{2}$ feature is the saturated Fraunhofer A-band at $0.76 \mu \mathrm{m}$ that is still relatively strong for significantly smaller mixing ratios than present Earth's. $O_{3}$ has two broad features, the extremely strong Huggins band in the UV shortward of $0.33 \mu \mathrm{m}$ and the Chappius band which shows as a broad triangular dip in the middle of the visible spectrum from about $0.45 \mu \mathrm{m}$ to $0.74 \mu \mathrm{m}$. Methane at present terrestrial abundance $(1.65 \mathrm{ppm})$ has no significant visible absorption feature, but at high abundance it has strong visible bands at $0.88 \mu \mathrm{m}$ and $1.0 \mu \mathrm{m}$, readily detectable in early Earth's history. $\mathrm{CO}_{2}$ has negligible visible features at present abundance, but in a high $\mathrm{CO}_{2}$-atmosphere of $10 \%$ it would have a significant band at $1.2 \mu \mathrm{m}$ and even stronger ones at longer wavelengths. Especially in the early evolution stage, the weak $1.06 \mu \mathrm{m}$ band can be observed (epoch 0 ).

\section{Conclusions}

Concentrating on the evolution of our planet we established a model for its atmosphere and the detectable biomarkers over its evolution history and showed the results for 
TPF-I/Darwin and TPF-C. These missions should be able to constrain an Earth-like planet in its evolution. Our calculations show that the atmospheric and the surface composition (especially in the visible) and cloud coverage influence the detected signal considerably (see Figure 1). From the 4 surfaces plotted on the left one sees that e.g. an Earth-size ocean planet will be more difficult to detect than a snow covered planet due to its low albedo (here we consider no clouds). The atmospheric features on an Earth-like planet change considerably over its evolution from a current day atmosphere (epoch 5 $=$ present Earth) over a $\mathrm{CO}_{2} / \mathrm{CH}_{4}$-rich atmosphere (epoch 3 around $2 \mathrm{Ga}$ ) to a $\mathrm{CO}_{2}$ rich $(3.9 \mathrm{Ga}=$ epoch 0$)$ see Figure 2. Atmospheric features on Earth can provide clues of biological activities for at least 2 billion years. $O_{3}$ shows a strong feature in the IR for the last $2 \mathrm{Ga}$ of Earth's history even at a low resolution of 25. $\mathrm{O}_{2}$ shows a weaker feature in the visible for the last $2 \mathrm{Ga}$ of Earth's history at a resolution of 70 (note that no noise level is assumed for these calculations). Methane (a potential bioindicator for early Earth) and nitrous oxide (a biomarker with a weak signature in the IR) have features nearly overlapping in the $7 \mu \mathrm{m}$ region, lying in the red wing of the $6 \mu \mathrm{m}$ water band. The absorption bands of those three species are different. It is not readily separable for current Earth at low spectral resolution. $\mathrm{N}_{2} \mathrm{O}$ is a very interesting molecule because it is produced in abundance by life but only in trace amounts by natural processes. It is unlikely to become a prime target for the first generation of space-based missions searching for extrasolar planets that will work with low resolution, but it is an excellent target for follow up missions. But if methane is abundant, as it probably was for early Earth, then it is readily detectable, even at low spectral resolution, functioning as an early bio-indicator for young Earth-like planets.

\section{Acknowledgements}

Special Thanks to J. Kasting, A. Knoll and A. Segura for constructive discussions.

\section{References}

Borde, P. \& Traub, W. 2005 IAUC200 proceedings

Charbonneau, D., Allen, L.E., Megeath, S.T., et al. 2005 ApJ 626

Charbonneau, D., Brown, T.M., Noyes, R.W., \& Gilliland, R.L. 2002 ApJ 568

Deming D., Seager S., Richardson L.J., Harrington J. et al. 2005 Nature 434

Des Marais, D.J., Harwit, M.O., Jucks, K.W. Kasting, J.F. et al. 2002 Astrobiology2

Ehrenfreund P. \& Charnley S.B. 2003 ESA SP-539

Fridlund, M., Kaltenegger, L. et al. 2006 in prep

Kaltenegger, L., Traub, W. \& Jucks, K. 2005 in prep

Kaltenegger, L., Fridlund, M.\& Karlsson, A. 2005 IAUC200 proceedings

Kasting, J.F. \& Catling D. 2003 Annual Rev. Aston. Astrophys. 41

Kasting, J.F. \& Siefert, J.L. 2002 Science 296

Kasting, J.F., Whitmire, D.P. \& Reynolds, R.T. 1993 Icarus 101,108

Meadows, V. et al. 2005 IAUC200 proceedings

Owen, T. 1980 Strategies for Search for Life in the Universe pp. 177

Pavlov, A.A., Hurtgen, M.T., Kasting, J.F., \& Arthur, M.A. 2003 Geology 31, pp. 87

Schopf, J.W. 1993 Science 260, pp. 460

Seager, S. 2002 astro-ph/0212550

Segura, A., Krelove, K., Kasting, J. et al. Astrobiology V.3, 4

Selsis, F., Despois, D. \& Parisot, J.-P. 2002 AA 388

Traub, W. \& Jucks, K. 2002 AGU 130, pp369

Turnbull, M., Traub, W., Jucks, K. et al. 2005 ApJ submitted

Woolf, N.J., Smith, P.S., Traub, W.A., \& Jucks, K.W. 2002 ApJ 574, pp430 